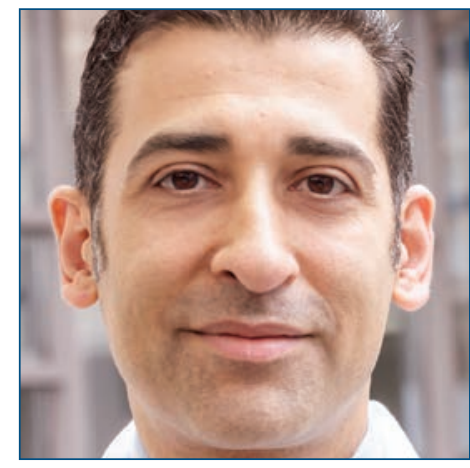

CHADI NABHAN, MD, MBA

Chadi Nabhan, MD, MBA, is a Vice President and Chief Medical Officer at Cardinal Health Specialty Solutions, where he works across all divisions of specialty solutions, providing resources to pharmaceutical companies, providers, payers, patients, and policy makers. In addition, Dr. Nabhan is engaged in data analytics, strategic initiatives, marketing communications, regulatory, and logistical initiatives in a value-based environment and a rapidly changing landscape of healthcare delivery.

Prior to joining Cardinal Health, Dr. Nabhan was the Medical Director of the Clinical Cancer Center at the University of Chicago, where he oversaw the clinical operations of cancer clinics supervising medical, surgical, and radiation oncology. He was instrumental in obtaining the QOPI certification for the University of Chicago and an integral member of the team implementing clinical care pathways.

Dr. Nabhan is a hematologist and medical oncologist with special interest in malignant hematology (leukemias and lymphomas) and genitourinary malignancies. He has been the primary investigator on many clinical trials, and some of his research has been presented at national and international meetings. He also has an interest in double-hit lymphomas and served on the Double Hit Lymphoma Foundation Board. Dr. Nabhan has more than 300 peer-reviewed articles, abstracts, and book chapters and serves on editorial boards of multiple scientific journals, including JAMA Oncology. $\mathrm{He}$ is also a reviewer for major medical journals. Dr. Nabhan is interested in developing strategies to improve cancer care delivery in an era where reimbursement models are rapidly changing; he has a focus on the business and economic aspect of oncology care as they impact all stakeholders.

doi: 10.6004/jnccn.2018.7092

The ideas and viewpoints expressed in this commentary are those of the author and do not necessarily represent any policy, position, or program of NCCN.

\section{Value in Oncology: It Is in the Eyes of the Beholder}

\author{
Chadi Nabhan, MD, MBA; Eli G. Phillips Jr, PharmD, JD; \\ and Bruce A. Feinberg, DO
}

$\mathbf{H}$ ealthcare expenditures in the United States are mounting at near double the rate of the consumer price index, with drug costs increasing faster than other contributors. ${ }^{1}$ The cost of most new anticancer therapies exceeds $\$ 10,000$ per month, regardless of whether there are competing drugs of the same class or for the same indication. ${ }^{2}$ New market entrants have neither curbed rising costs nor led to improved outcomes for patients in the United States compared with other industrialized nations. ${ }^{3}$ This disconnect between higher prices, increased competition, and limited clinical benefits has challenged the historical basis for measuring value of cancer drugs using the parameters of efficacy and toxicity (safety) rather than quality and cost. Today, value is more often defined as the balance between desired patient outcomes and the costs incurred to achieve these outcomes. ${ }^{4}$

More than a decade ago, the Institute of Medicine (IOM) challenged the notion of a 2-parameter basis for value in healthcare by establishing 6 core components. ${ }^{5}$ Professional societies and advocacy groups have inconsistently adopted the IOM definitions as they developed value-assessment frameworks in an attempt to improve shared decision-making. ${ }^{6}$ The ASCO Value Framework, ${ }^{7}$ NCCN Evidence Blocks, ${ }^{8}$ ESMO Magnitude of Clinical Benefit Scale (MCBS), ${ }^{9}$ and the Institute for Clinical and Economic Review (ICER) ${ }^{10}$ are examples of how large organizations are tackling the value-based healthcare debate. As the dialogue progresses, it is becoming increasingly apparent that various stakeholders see value through very different prisms. We assess how value perspectives for cancer drugs differ among the stakeholders (payers, manufacturers, providers, patients) and propose an approach based on a unifying value principle espoused by all stakeholders: administer the right drug to the right patient at the right time.

\section{Value to Payers}

By virtue of benefit design, commercial insurance companies dictate, to some degree, where, when, and what care is received. They also dictate, to some degree, who administers the care, reimbursement to the treating providers, and out-ofpocket costs to the patient. Those levers of control over the cancer care plan are often perceived as limiting patient choice and physician autonomy. Insurers argue that these restrictions are checked and balanced by appeal processes and medical policies that define what is medically necessary and not experimental based on best available evidence (including FDA label and NCCN Guidelines). The fact that neither the FDA nor NCCN directly include cost in their respective determination of approval or inclusion places payers in the uncomfortable position of "being the adult in the room" and having to say "no" if excessive cost shifts the value determination from green to red. But this "virtuous" position is challenged by a financial model based on dollars not spent on healthcare, curiously named "medical loss ratio." 11 Payers' perception of "the right drug for the right patient at the right time" is the most weighted toward cost and is consistent with their perceived role as stewards of the healthcare dollar. The payer value perspective and the tools used to support it that restrict access, choice, and autonomy make it least aligned with other stakeholders.

\section{Value for Manufacturers}

Although payers project their position of stewardship over the healthcare dollar as virtuous, manufacturers project a similar nobility for their role as innovators 
pragmatically defined by intellectual property (IP) rights or patents. Manufacturers spend significant resources trying to extend patent life because the protections patents afford will eventually expire. This allows unfettered development of competitor products. Therefore, manufacturers require a consistent pipeline of new and innovative products to achieve sustainability and a competitive edge attained through research and development of new products, new indications, or acquisition of the IP of another manufacturer. Because each of these initiatives requires significant capital investment, manufacturers need successful sales of current products to fund the future successes of the company. The commercial success of a drug, and therefore value to a manufacturer, is highly dependent on price and patient access. This in turn depends on providers' clinical adoption-with influence from payers-but clinical adoption is not possible without FDA approval.

Gaining the endorsements of multiple stakeholders requires some market differentiation, such as improved outcomes, decreased side effects, lower costs, or a mechanism that could lead to increased adherence, such as a simplified form of administration. If a product demonstrates one of these advantages through comparative effectiveness studies, it may qualify for better positioning on formularies, which can lead to further increases in clinical adoption rates. The manufacturer perspective of cancer drug value thereby incorporates the complexity and cost of innovation, the financial risks inherent in "orphan disease" (as some cancers are) drug development, and measurable benefit in quality and length of life. It is incumbent upon a manufacturer to prove differentiation before it can assert that its product is "the right drug for the right patient at the right time."

\section{Value to Providers}

The Darwinian notion that healthcare providers, especially those in community practice, are economic players acting primarily in their own financial best interest has been roundly challenged. ${ }^{12}$ The behavioral economics of physician prescribing is remarkably complex, because innumerable biases influence decisionmaking, especially with many new entrants in the healthcare market. ${ }^{13,14}$ As complex as the concept of value may be in healthcare-and particularly cancer care-that complexity is unparalleled when considering providers. The complexity by which healthcare providers appreciate value can include their patients' clinical outcomes, experience, and financial outcomes; their own professional satisfaction; their practice's (business) success; and, increasingly, societal expectations. These myriad issues are often at odds; they are both clinical and objective, intuitive and subjective, pragmatic and operational, mundane and financial.

Physicians make treatment determinations for each clinical scenario by balancing clinical outcomes (eg, efficacy of a particular therapy, its expected and possible unexpected toxicities, and effect on a patient's quality of life) with the financial impact to the patient, practice, and society. Such determinations are further informed by payer policy, which may include clinical pathways, bundled or episode care reimbursement in commercial plans, or participation in the Oncology Care Model, with its inherent reporting requirements and reimbursement challenges. ${ }^{15}$

Among this matrix of factors influencing physician determination of value, none is more important than published clinical outcomes. In cancer care, these can be complex and highly variable. They are often not representative of real-world patients and can depend on clinical scenarios. The interdisciplinary nature of how many cancers are treated adds to the complexity of defining appropriate outcome measures. Although some metrics are common between various payers and regulatory bodies, consistent variations exist as well. Mullangi et al ${ }^{16}$ highlighted significant variations in outcome measures between several quality programs in colon, prostate, lung, and breast cancers. Provider perspectives are heavily informed by their role as caregivers entrusted with the lives of patients who are dealing with

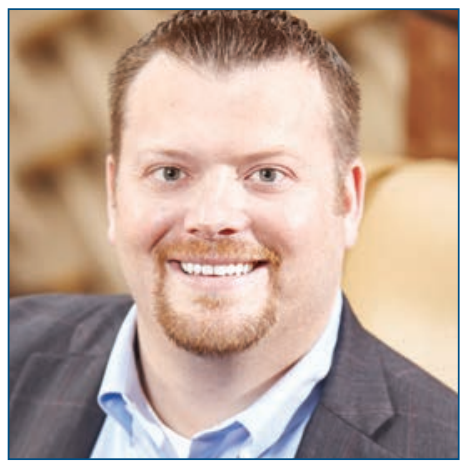

ELI G. PHILLIPS JR, PharmD, JD

Eli G. Phillips Jr, PharmD, JD, is a licensed pharmacist and attorney who joined Cardinal Health in June 2014. He currently serves as Vice President for Insights \& Engagement within Cardinal Health Specialty Solutions, which provides a wide range of services for the pharmaceutical industry, including: health economics and outcomes research, synthesis and analysis of real world evidence, and commercial marketing through provider engagement.

He has previously held a variety of leadership positions, most recently serving as the head of Quality and Regulatory Affairs for each of the enterprise's Specialty Businesses. Before joining Cardinal Health, Dr. Phillips served as a professor in both pharmacy and law schoo settings and served as Managing Member of Healthcare Advising, LLC; a private regulatory consulting and data analytics firm.

Dr. Phillips completed his pharmacy studies at Wilkes University and legal studies at Drexel University, where he earned certificates in Health Law and Intellectual Property Law. He currently serves as a member of the board of directors for Adaptive Sports Connection, a nonprofit organization whose mission is to empower children, adults, and veterans with physical and cognitive challenges through sports and therapeutic outdoor recreation. 


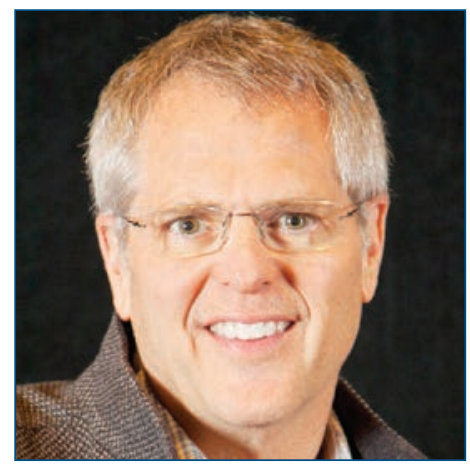

BRUCE A. FEINBERG, DO

Bruce A. Feinberg, DO, serves as Vice President of Clinical Affairs and Chief Medical Officer for Cardinal Health Specialty Solutions. He works across the specialty solutions businesses, consulting with payers, providers, pharma, patients, and policy makers. Dr. Feinberg has played a vital role in the nascent value-based healthcare movement and was instrumental in developing PathWare, leading decision support and transaction software. Since joining Cardinal Health, Dr. Feinberg has made more than 70 peer-reviewed contributions to value-based healthcare literature. In 2015, Dr. Feinberg was honored as a PharmaVoice 100 most inspirational life-science leaders.

Dr. Feinberg is the author of bestselling books and has been consulted by prominent national media outlets, including The New York Times, The Wall Street Journal, Forbes, and CNN. As host of The Weekly Check-Up on WSB Radio in Atlanta, he provides the community the latest health information and an opportunity to express their views on health and medicine.

Prior to joining Cardinal Health, Dr. Feinberg was the founder and CEO of Georgia Cancer Specialists (GCS), one of the first and largest integrated oncology specialty practices in the United States. At GCS, he pioneered health information technology, the pathways movement, and the oncology medical home, publishing the first report of their impact on financial and clinical outcomes in 1998. chronic, disabling, and life-threatening diseases, but these perspectives are also biased by the economic environment in which providers practice. Providers may perceive themselves as pawns on a healthcare chessboard in which payers and manufacturers battle for dominance at clinicians' expense. Providers often appear to have less interest in the specifics of the debate; rather, they may interpret value as clarity, stability, and fairness in the rules of engagement within the healthcare marketplace. Providers may perceive "the right drug for the right patient at the right time" as an oversimplified maxim to a highly complex problem.

\section{Value for Patients}

For most patients immediately confronted with a life-threatening disease, value has a simple translation: cure. The instinct for survival is so strong that rational decision-making can be impaired, with comprehension of facts distorted, therapeutic benefits exaggerated, and risks ignored. "Value" as perceived by patients with life-threatening diseases often changes as they progress through the 5 stages of grief defined by Elisabeth Kübler-Ross. ${ }^{17}$ Value perceptions will also be significantly influenced by patients' age, comorbidity, prognosis, support system, and financial position, and nature and extent of recommended treatment. Other end points that patients may value include ability to retain employment, sexual function, the impact of cancer and survivorship on family and caregivers, and grade 2 toxicities that are often not mentioned but have a detrimental impact on quality of life. Current clinical trials and cancer value calculators often do not address these essential items, which affect value to patients. ${ }^{6}$ Observational and registry studies attempt to answer some of these questions, but the output from these analyses is less robust.

A patient-centered approach to medicine has led to the increasing use of shared decision-making, a process by which physicians engage patients to actively participate in their care plan. Such participation requires an understanding of the value of the benefits garnered from a therapy and the potential toxicities that may impact length or quality of life. Value calculators have not been compared headto-head. When an attempt was made to compare the value frameworks of ASCO and ESMO, correlation was absent. This underscores the need to develop future frameworks with patient voice and needs in mind. ${ }^{1}$

Duration and extent of benefit is a critical end point for most patients; however, this benefit does not always correlate with traditional progression-free survival. In clinical trials, progression may be defined arbitrarily based on target lesion measurement changes without accounting for true clinical benefit. ${ }^{18}$ In fact, recognizing that clinical benefit and radiographic changes are not always aligned led to the suggestion that new immunotherapies improved patient outcomes even when initial progression was noted. Such nuances raise concerns that the complexity of clinical evidence and the limitations of health literacy might impair shared decision-making and compromise the contribution of patients' perception of value in the ongoing debate. Right to try legislation might be seen as a consequence of these realities despite the well-known limitations of and opposition to this law. ${ }^{19,20}$ Although the influences are many, the core principle in the patient perspective of "the right drug at the right time" appears heavily weighted to unrestricted access.

\section{The Path Forward}

The apparently unsolvable riddle of value has resulted in interesting new contract models piloted to bridge stakeholder conflicts through risk-sharing. Payers and providers have used new methods of engagement: bundled payments, episodes of care, capitation, patient-centered homes, and accountable care organizations. Payers and manufacturers are experimenting with contractual arrangements based on outcome metrics rather than the historical negotiation for better contract terms (rebates) often conducted via a contracted pharmacy benefit manager. In these 
new arrangements, tradeoffs are made between access and cost with the hope of producing mutually beneficial financial outcomes. Even in such provocative models, it is difficult to design inclusion of all stakeholders, and patients and providers often feel left out. Recent declarations by major insurers that they will pass savings on to patients offer hope of greater inclusion and transparency, but the devil will be in the details.

Consensus among stakeholders regarding who is the right patient, which is the right drug, and when is the right time is unlikely to come soon enough. This question begs for third-party mediation. Although the United Kingdom solution of empowering its National Institute for Health and Care Excellence (NICE) is uniformly vilified by stakeholders in the United States, it effectively tackles this thorny problem across the pond. Whether advocacy organizations like NCCN or ASCO can serve in this capacity, nonprofit institutes can be de facto arbiters of value, or the FDA or other federal agency will step up remains to be seen. That a market solution is not readily available is clear, because any such solution that categorically defines the right patient, the right drug, and the right time will require a declaration of winners and losers.
Manufacturers will lose when their drug is not chosen; payers will lose when draconian measures that create barriers to access are obviated by consensus standards; providers will lose via restriction of autonomy; and patients will lose via limitations of choice. This combination of outcomes seems culturally challenging in the United States.

To move into a true value-based care environment, all stakeholders involved must agree on uniform definitions. To that end, every stakeholder must critically evaluate whether their strategy and decision-making benefit patients and their caregivers while preserving patient choice and respecting physician autonomy. Although profitable growth leads to further investments in innovation and has generated impressive advances in the current state of the art, the more patients benefit, the more every party involved in the healthcare ecosystem will benefit. If the right patient receives the right drug at the right time, all stakeholders will experience the sought-after value. Eliminating barriers between these stakeholders and starting an honest dialogue on how to define value and how to engage patients is a small, but very important, step forward.

\section{References}

1. Evans WK, Cheung MC, Chan KK. Measuring value and benefit: a matter of perspective. Lancet Oncol 2017;18:839-840.

2. Kantarjian HM, Fojo T, Mathisen $M$, et al. Cancer drugs in the United States: Justum Pretium—the just price. J Clin Oncol 2013;31:3600-3604.

3. Ridic G, Gleason S, Ridic O. Comparisons of health care systems in the United States, Germany and Canada. Mater Sociomed 2012;24:112-120.

4. Porter ME. What is value in health care? N Engl J Med 2010;363:24772481.

5. Institue of Medicine Committee on Quality of Health Care in America. Crossing the Quality Chasm: A New Health System for the 21st Century. Washington, DC: National Academies Press; 2001.

6. Nabhan C, Feinberg BA. Value-based calculators in cancer: current state and challenges. J Oncol Pract 2017;13:499-506.

7. Schnipper LE, Davidson NE, Wollins DS, et al. Updating the American Society of Clinical Oncology Value Framework: revisions and reflections in response to comments received. J Clin Oncol 2016;34:2925-2934.

8. Carlson RW, Jonasch E. NCCN Evidence Blocks. J Natl Compr Canc Netw 2016;14:616-619.

9. Cherny NI, Sullivan R, Dafni U, et al. A standardised, generic, validated approach to stratify the magnitude of clinical benefit that can be anticipated from anti-cancer therapies: the European Society for Medical Oncology Magnitude of Clinical Benefit Scale (ESMO-MCBS). Ann Oncol 2015;26:1547-1573; erratum in: Ann Oncol 2017;28:2901-2905.

10. Pearson SD. The ICER Value Framework: integrating cost effectiveness and affordability in the assessment of health care value. Value Health 2018;21:258-265.
11. Karaca-Mandic P, Abraham JM, Simon K. Is the medical loss ratio a good target measure for regulation in the individual market for health insurance? Health Econ 2015;24:55-74.

12. Feinberg B. Real-world evidence and the behavioral economics of physician prescribing. Am J Manag Care 2017;23:254-256.

13. Khullar D, Chokshi DA, Kocher R, et al. Behavioral economics and physician compensation-promise and challenges. N Engl J Med 2015;372:2281-2283.

14. Nabhan C, Feinberg BA. Behavioral economics and the future of biosimilars. J Natl Compr Canc Netw 2017;15:1449-1451.

15. Nabhan $C$, Jeune-Smith $Y$, Klinefelter $P$, et al. Challenges, perceptions, and readiness of oncology clinicians for the MACRA quality payment program. JAMA Oncol 2018;4:252-253.

16. Mullangi S, Schleicher S, Feeley TW. Outcome measurement in value-based payments. JAMA Oncol 2017;3:1019-1020.

17. Smaldone MC, Uzzo RG. The Kubler-Ross model, physician distress, and performance reporting. Nat Rev Urol 2013;10:425-428.

18. Kemp R, Prasad V. Surrogate endpoints in oncology: when are they acceptable for regulatory and clinical decisions, and are they currently overused? BMC Med 2017;15:134.

19. Kirkner RM. Can Congress get 'right to try' right? Manag Care 2018;27:10-11.

20. Bateman-House A, Robertson CT. The Federal Right to Try Act of 2017: a wrong turn for access to investigational drugs and the path forward. JAMA Intern Med 2018;178:321-322. 